\title{
Localized Subharmonic Waves in a Circularly Vibrated Granular Bed
}

\author{
Andreas Götzendorfer, Christof A. Kruelle, and Ingo Rehberg \\ Experimentalphysik V, Universität Bayreuth, D-95440 Bayreuth, Germany \\ Daniel Svenšek \\ Department of Physics, University of Ljubljana, Jadranska 19, SI-1000 Ljubljana, Slovenia and Theoretische Physik III, \\ Universität Bayreuth, D-95440 Bayreuth, Germany \\ (Received 15 March 2006; published 10 November 2006)
}

\begin{abstract}
Localized period-doubling waves arise in circularly shaken granular beds contained in an annular channel. These solitary wave packets are accompanied by a locally increased particle density. The width and velocity of the granular wave pulse are measured as a function of the bed height. A continuum model for the material distribution, based on the measured granular transport velocity as a function of the bed thickness, captures the essence of the experimental findings.
\end{abstract}

DOI: 10.1103/PhysRevLett.97.198001

La Ola, the Mexican wave [1] serves as a good illustration for solitary waves in an annular system. On a smaller scale, the confined state in binary mixture convection [2] and "worms" in electroconvection [3] are prime examples of solitary waves in dissipative systems, featuring moving pulses of traveling waves. Moreover, periodically driven systems are known to exhibit highly localized stationary structures, coined oscillons [4], or propagating solitary waves with the periodicity of the driving [5]. Here we present drifting localized pulses that envelop parametrically driven, subharmonic, standing waves. They arise in a circularly vibrated granular bed.

The granular material was contained in a $2 \mathrm{~cm}$ wide and $7 \mathrm{~cm}$ high annular channel with radius $R=22.5 \mathrm{~cm}$ and circumference $L=141 \mathrm{~cm}$. The side walls were made of transparent Plexiglas mounted on a stainless steel floor. The vertical component of the container oscillation was $z(t)=A_{v} \sin (2 \pi f t)$ with an amplitude $A_{v}$ of $2.06 \mathrm{~mm}$ at a frequency $f$ of $26.0 \mathrm{~Hz}$. Additionally, the ring performed an angular oscillation $\alpha(t)=\left(A_{h} / R\right) \sin (2 \pi f t-\pi / 2)$ at the same frequency around its axis of symmetry. The horizontal oscillation amplitude in the center of the channel $A_{h}$ was $1.87 \mathrm{~mm}$. Accordingly, every point of the channel floor followed approximately a circular trajectory in a plane tangent to the ring. A more detailed description of the experimental setup and the driving mechanism is given in Refs. [6,7].

We use spherical basalt glass beads (density: $3.0 \mathrm{~g} / \mathrm{cm}^{3}$ ) with diameter $d=1.18 \pm 0.03 \mathrm{~mm}$. The amount of material in the container is given by the number of particle layers $H_{0}$ that form when the material is evenly distributed around the ring and the particles are close packed. A densely packed monolayer consists of 21500 particles. In the experiments $H_{0}$ was varied in steps of 0.5 .

The granular system was observed from the top via a conical mirror placed in the center of the ring. Thus a side view of the whole channel was captured with a single highspeed digital camera (resolution: $1280 \times 1024$ pixels at 500 frames per second). For stroboscopic recordings the
PACS numbers: 45.70.Qj, 47.57.Gc

camera is triggered externally. Image processing delivers $360^{\circ}$ panoramic side views of the granular profile in the channel.

For $H_{0} \leq 8.5$ or $H_{0} \geq 15.5$ the uniform material distribution is stable. In both cases manually generated heaps and holes decay within seconds. Shallow beds $\left(H_{0} \leq 8.5\right)$ are flat and oscillate at the forcing frequency. Deep beds $\left(H_{0} \geq 15.5\right)$ exhibit subharmonic waves with constant envelope throughout the whole channel [8]. Within the intermediate regime, $8.5<H_{0}<15.5$, the uniform material distribution is unstable for $H_{0}<12.0$, decaying spontaneously into regions of decreased bed thickness that are flat and oscillate at the forcing frequency and regions of increased bed thickness where standing waves are present. After a coarsening process of about 1 min regions of increased bed thickness have merged into a single pulse of subharmonic waves (see Fig. 1). Bistability of the uniform material distribution and the pulse state is ob-

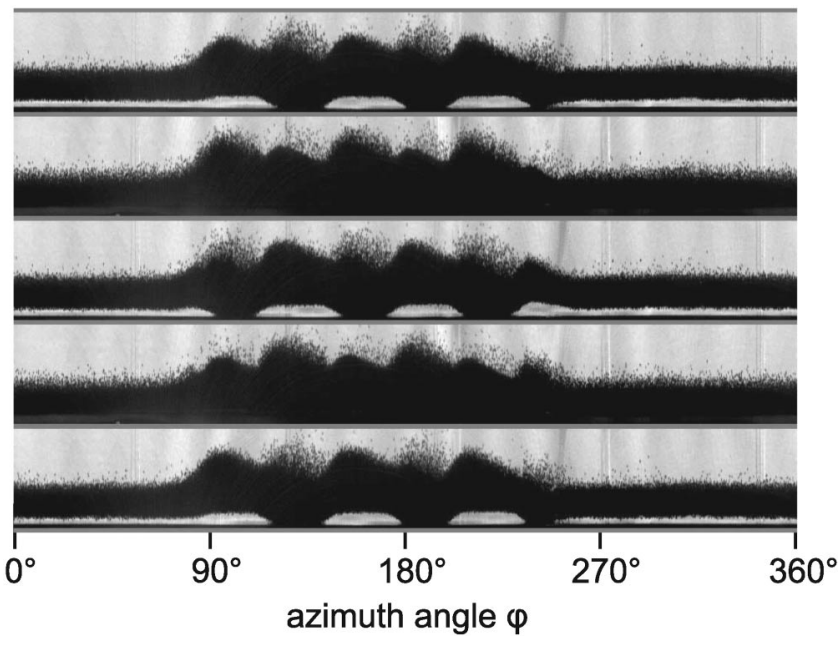

FIG. 1. Panoramic snapshots through the inner sidewall of the channel. Time increases from top to bottom in steps of half a forcing period. For clarity, the images are stretched vertically by a factor of 3.5. $H_{0}=12.0$. 
served for $12.0 \leq H_{0}<15.5$. In this range either extended or localized waves are observed. To check whether the pulse is also present under pure vertical forcing the horizontal oscillation component was turned off by changing the machine settings. In consequence, uneven material distributions became unstable and particle diffusion led to an equal bed thickness in the whole container.

In order to elucidate the spatiotemporal dynamics of the bed undulations we plot the gap width between the container floor and the layer bottom in gray scale as a function of azimuth angle and time. The side views are divided into 1024 vertical stripes ranging from the bottom of the container to the middle of the bed. The mean brightness of each stripe is then taken as a measure of the local gap width.

Figure 2 depicts the gap width for the situation of Fig. 1: Outside the pulse the gap width is constant in space and the layer detaches from the floor every forcing cycle. Within the pulse the layer is undulated and the oscillation frequency is half the forcing frequency. Both the wave pattern and the pulse drift to the left, yet at a different velocity, the pulse being about 2 times faster than the waves. This implies that new waves are constantly created at the leading front of the pulse whereas the last wave in the pulse is shrinking. As soon as the last wave falls below a critical size it detaches from the pulse, slows down, and even reverses its direction of movement until it dissolves completely.

To separate the slow dynamics of the pulse envelope from the rapid oscillations of the substructure one image per forcing cycle was recorded at a fixed phase. Since the

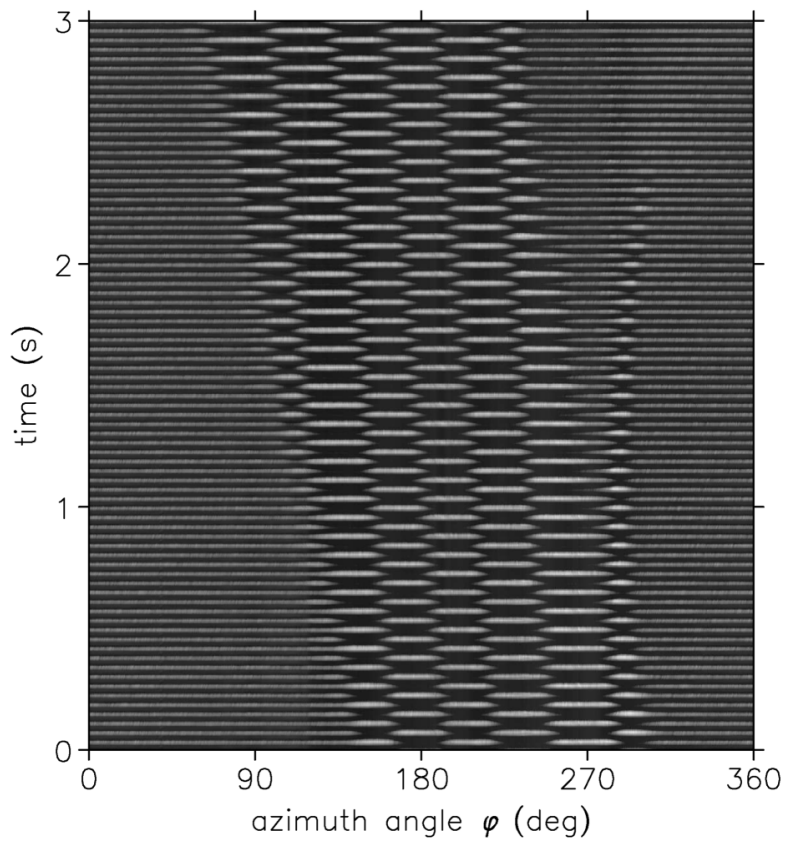

FIG. 2. Width of the gap between the container floor and the layer bottom as a function of azimuth angle $\varphi$ and time, coded in gray scale. The brightest regions correspond to a gap width of approximately $10 \mathrm{~d}$. period doubling is not of interest here we average two successive images. A measure for the bed extension, as depicted in Fig. 3(b), was obtained by a technique similar to the one used to extract the gap width. The major difference was that here we calculated the mean brightness of vertical stripes that cover the whole image instead of only the lower part. The space-time plot shows a pulse with constant length and velocity performing several revolutions in the annular container. Positions of leading and trailing fronts are determined by largest gradients. The pulse velocity is determined by fitting straight lines through the detected positions.

To shed light on the mechanism behind the phenomenon of localized pattern formation, we determined the transport velocity $v_{t}\left(H_{0}\right)$, defined for steady states with uniform material distribution as the particle velocity along the channel averaged over all particles and many forcing cycles. In fact we measured the time that a large $(d=$ $1 \mathrm{~cm}$ ) wooden tracer particle needed to travel a certain distance. By visual inspection we conclude that the mean tracer velocity is representative for the transport velocity. This is also corroborated by experiments in a linear channel where the transport velocity, determined by simultaneous measurement of the mass flow and the total amount of material in the trough, was compared to the velocity of tracer particles [9]. The tracer particle is subject to Brownian motion with a diffusion constant of about $1 \mathrm{~cm}^{2} / \mathrm{s}$. This value was extracted from the deviations from the mean time the particle needs to perform one revolution in the annular channel. Total observation times exceeded $200 \mathrm{~s}$ in order to keep the error for the transport velocity below $0.1 \mathrm{~cm} / \mathrm{s}$.

In region I of Fig. 4 the transport velocity is positive (in the direction of increasing angle $\varphi$ ) and grows linearly with $H_{0}$. The uniform material distribution is unstable in region II, preventing the determination of the transport

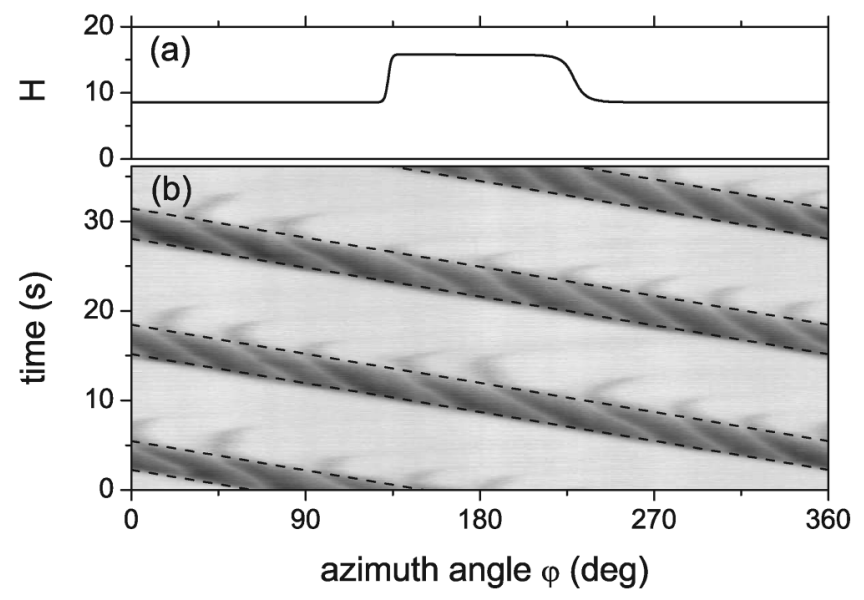

FIG. 3. Traveling pulse observed at $H_{0}=10.5$. (a) Local bed height in the steady state as obtained from the model. (b) Measured vertical bed extension as a function of azimuth angle $\varphi$ and time, coded in gray scale. The darker the thicker is the bed. Broken lines indicate linear fits to the front positions. 


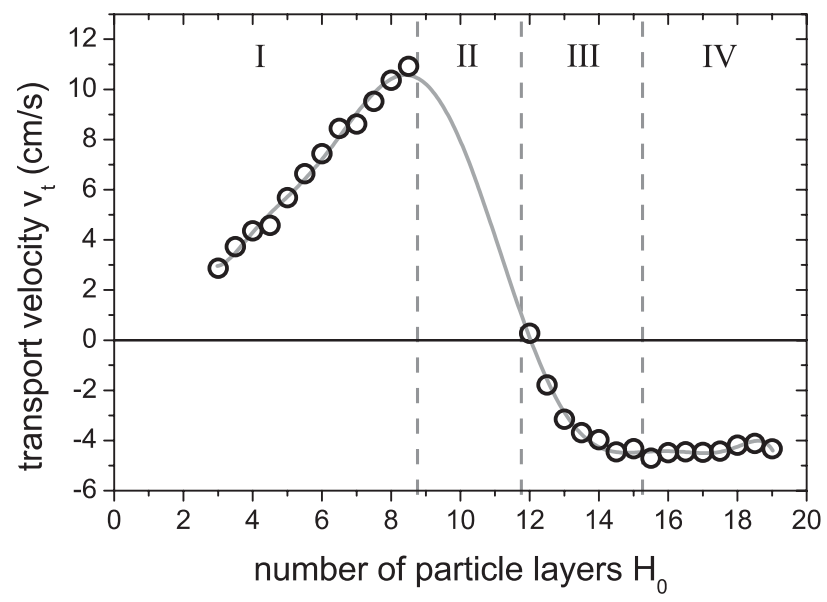

FIG. 4. Transport velocity $v_{t}$ of the granular material as a function of the number of particle layers $H_{0}$. The curve represents a 9th order polynomial fit used in the model.

velocity in this range. In regions III and IV the transport velocity decreases, becomes even negative, and finally attains a constant value. In the latter regions subharmonic waves are present that drift with the same velocity as the tracer particle. The different transport behavior below and above region II is due to the transition from periodic to period-doubling dynamics. Beds consisting of less than 8.5 particle layers are in contact with the container floor at different intervals of the driving period than beds with more than 12.0 particle layers. Thus the local bed thickness via the corresponding oscillation dynamics affects the local transport velocity $[10,11]$, which in turn leads to a change in local bed thickness.

To conceive this mechanism, we adopted a onedimensional continuum model for the granular flow. The measured bed-height dependent transport velocity was taken as input, while details of the granular dynamics were neglected. We introduce a local bed height $H(x, t)$, where $x$ denotes the position along the ring and $t$ the time, which satisfies $\int H(x, t) d x=H_{0} L$. The continuity equation for this local bed thickness reads

$$
\frac{\partial H}{\partial t}+\frac{\partial(H v)}{\partial x}=0
$$

where $v(x, t)$ is the local flow velocity. The analogue of the Navier-Stokes equation can be written as

$$
\frac{\partial v}{\partial t}+v \frac{\partial v}{\partial x}=-\frac{1}{H} \frac{\partial p}{\partial x}+\frac{f_{e}}{H}+\frac{1}{H} \frac{\partial}{\partial x}\left(\mu \frac{\partial v}{\partial x}\right) .
$$

For $p$, the equivalent of the pressure, we took $p=c_{0}^{2} \cdot H$ with constant $c_{0}$ in order to model the tendency of the particles to move against the local bed-height gradient. The analogue of the force density is given by

$$
f_{e}=H \frac{v_{t}(H)-v}{\tau} .
$$

This ansatz implies that for uniform material distributions the granulate attains the velocity $v_{t}(H)$ by an exponential relaxation process with a time constant $\tau$. To avoid numerical artifacts connected with kinks we assume $v_{t}(H)$ to be a smooth function and approximate the experimental transport velocity data by a polynomial (see Fig. 4). The analogue of the viscosity $\mu$ is assumed to be constant. Equation (2) then becomes

$$
\frac{\partial v}{\partial t}+v \frac{\partial v}{\partial x}=-\frac{c_{0}^{2}}{H} \frac{\partial H}{\partial x}+\frac{v_{t}(H)-v}{\tau}+\frac{\mu}{H} \frac{\partial^{2} v}{\partial x^{2}} .
$$

Depending on the parameters, the steady-state solution of the model (1) and (3) is either homogeneous flow or a traveling pulse of fixed shape [Fig. 3(a)]. Linearizing Eqs. (1) and (3) around $H=H_{0}$, one finds that the homogeneous flow is stable against a perturbation with the wave vector $q=0$ if

$$
H_{0}\left|v_{t}^{\prime}\left(H_{0}\right)\right|<c_{0} .
$$

Since it has been shown that the instability first occurs for $q=0$ [12], if condition (4) is fulfilled, the flow is stable against any perturbation.

Guided by experimental observation we chose $\tau=$ $0.1 \mathrm{~s}$, corresponding to about three forcing cycles. Note that $\tau$ is small compared to the time it takes for the material to travel through the pulse. In this limit, the pressure and diffusion terms in Eq. (3) can be neglected everywhere except at the fronts, and the pulses exhibit a plateau where $v=v_{t}(H)$ [Fig. 3(a)]. We verified that in this limit the results are insensitive to $\tau$. The viscous term was kept just large enough to stabilize the leading front and sufficiently small not to influence significantly the results. A value $\mu=$ $50 \mathrm{~cm}^{2} / \mathrm{s}$ has been chosen, resulting in a rather steep leading front which corresponds to the experimental observation (see Fig. 1). The only vital parameter of the model is thus $c_{0}$.

Increasing $H_{0}$ enlarges the pulse [see Fig. 5(b)]. The region of stability for the pulse solution obtained from the model matches the experiments best when choosing $c_{0}^{2}=$ $600 \mathrm{~cm}^{2} / \mathrm{s}^{2}$. Then the model gives stable homogeneous flow for $H_{0}<9.66$ or $H_{0}>13.18$, and a stable pulse for $8.75<H_{0}<15.32$. In case the homogeneous flow is unstable, several pulses, generated by small perturbations, coalesce to a single steady pulse on the time scale of a minute, in agreement with experiment. According to the model the pulse solution bifurcates subcritically at $H_{0}=$ 9.66 and $H_{0}=13.18$, respectively. Hence there exist two bistable regions. Given that only one parameter was adjusted, the match in pulse length between theory and experiment in the whole range of $H_{0}$ is remarkable. The pulse velocity $v_{p}$, displayed in Fig. 5(a), is almost constant amounting to about $-11 \mathrm{~cm} / \mathrm{s}$, except for the shortest pulse, which is approximately $30 \%$ slower. The model also gives an almost constant pulse velocity. Why it is a factor of 2 off remains an open question. Strikingly, the chosen value of $c_{0}=24 \mathrm{~cm} / \mathrm{s}$ is of the same order of magnitude as the container speed, which amounts to 


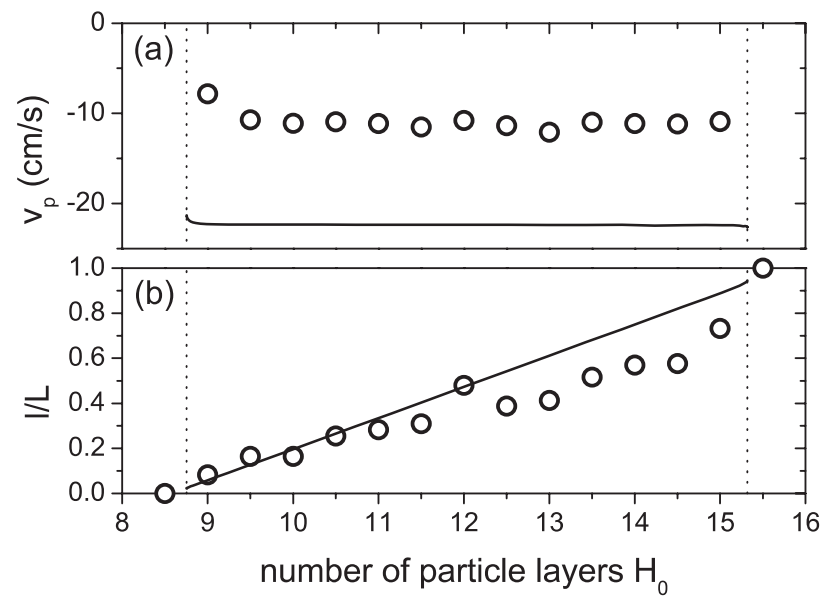

FIG. 5. (a) Measured pulse velocity $v_{p}$ and (b) relative pulse length $l / L$ as functions of the number of particle layers $H_{0}$, and comparison with the model (solid lines). Dotted lines demarcate the region of stability for pulses.

$32.6 \pm 1.1 \mathrm{~cm} / \mathrm{s}$ and can be taken as the typical speed transmitted to a particle upon collision with the container floor. The prefactor of the last term in Eq. (3) can be considered as the velocity diffusion constant. For beds consisting of more than ten particle layers it is comparable in magnitude to the tracer particle's diffusion constant. Another interesting aspect of the model is the fact that the bed heights inside and outside the pulse always differ by $\left|H_{2}-H_{1}\right|=7.2$ independent of $H_{0}$. Only the length of the pulse grows when material is added.

In conclusion, around the transition to period-doubling dynamics drastic changes in the transport velocity produce localized subharmonic wave packets in a circularly vibrated bed. Standing waves appear throughout a pulse of increased bed thickness. The pulse arises from a subcritical bifurcation and obtains a plateaulike shape. Its length increases when material is added while its velocity remains constant. As in our earlier experiments on the sublimation of a vibrated monolayer [6], two regions with different particle coverage coexist. However, in that case phase separation was still observed for pure vertical forcing when no material transport occurs.

Waves that appear if the local velocity in a onedimensional, compressible flow is a function of the local density are called kinematic waves [13]. One example are density waves observed in granular flows in a vertical pipe, where the sedimentation rate is a nonlinear function of the local particle density [14]. In the context of road traffic, the fundamental diagram describes the connection between flow rate and vehicle density [15]. Our ansatz, i.e., Eqs. (1) and (3) is inspired by a traffic flow model that explains the spontaneous appearance of pulses — "phantom jams" - out of initially homogeneous flow [12]. Plugging in the measured transport velocity as a function of bed thickness, our model for the dynamics of the mate- rial distribution retains the basic features of the pulses seen in the experiments.

The experiments were supported by Deutsche Forschungsgemeinschaft via the program "Verhalten granularer Medien". D. S. acknowledges support of the Slovenian Research Agency (No. Z1-7171-1554) and the Alexander von Humboldt Foundation.

[1] I. Farkas, D. Helbing, and T. Vicsek, Nature (London) 419, 131 (2002).

[2] P. Kolodner, D. Bensimon, and C. M. Surko, Phys. Rev. Lett. 60, 1723 (1988); K. E. Anderson and R. P. Behringer, Phys. Lett. A 145, 323 (1990); P. Kolodner, Phys. Rev. Lett. 66, 1165 (1991); W. Barten, M. Lücke, and M. Kamps, Phys. Rev. Lett. 66, 2621 (1991); H. Riecke, Phys. Rev. Lett. 68, 301 (1992); W. Barten, M. Lücke, M. Kamps, and R. Schmitz, Phys. Rev. E 51, 5662 (1995); D. Jung and M. Lücke, Phys. Rev. Lett. 89, 054502 (2002); J. J. Niemela, G. Ahlers, and D. S. Cannell, Phys. Rev. Lett. 64, 1365 (1990).

[3] M. Dennin, G. Ahlers, and D. S. Cannell, Phys. Rev. Lett. 77, 2475 (1996); H. Riecke and G. D. Granzow, Phys. Rev. Lett. 81, 333 (1998).

[4] P. Umbanhowar, F. Melo, and H. L. Swinney, Nature (London) 382, 793 (1996).

[5] O. Lioubashevski, H. Arbell, and J. Fineberg, Phys. Rev. Lett. 76, 3959 (1996); O. Lioubashevski, Y. Hamiel, A. Agnon, Z. Reches, and J. Fineberg, Phys. Rev. Lett. 83, 3190 (1999).

[6] A. Götzendorfer, J. Kreft, C. A. Kruelle, and I. Rehberg, Phys. Rev. Lett. 95, 135704 (2005).

[7] C. A. Kruelle, A. Götzendorfer, R. Grochowski, I. Rehberg, M. Rouijaa, and P. Walzel, in Traffic and Granular Flow '05, edited by A. Schadschneider, M. Schreckenberg, and D. Wolf (Springer, Berlin, 2006).

[8] M. Faraday, Phil. Trans. R. Soc. London 121, 299 (1831); S. Douady, S. Fauve, and C. Laroche, Europhys. Lett. 8, 621 (1989); F. Melo, P. Umbanhowar, and H. L. Swinney, Phys. Rev. Lett. 72, 172 (1994); C. Bizon, M. D. Shattuck, J. B. Swift, W. D. McCormick, and H. L. Swinney, Phys. Rev. Lett. 80, 57 (1998); O. Sano, Phys. Rev. E 72, 051302 (2005).

[9] R. Grochowski, S. Strugholtz, H. El hor, S. J. Linz, and P. Walzel, in PARTEC 2004, edited by S. E. Pratsinis, H. Schulz, R. Strobel, and C. Schreglmann (NürnbergMesse GmbH, Nuremberg, 2004).

[10] R. Grochowski, P. Walzel, M. Rouijaa, C. A. Kruelle, and I. Rehberg, Appl. Phys. Lett. 84, 1019 (2004).

[11] H. El Hor and S. J. Linz, J. Stat. Mech. 2005 L02005.

[12] B. S. Kerner and P. Konhäuser, Phys. Rev. E 48, R2335 (1993); D. A. Kurtze and D. C. Hong, Phys. Rev. E 52, 218 (1995).

[13] G. B. Whitham, Linear and Nonlinear Waves (Wiley, New York, 1974).

[14] O. Moriyama, N. Kuroiwa, M. Matsushita, and H. Hayakawa, Phys. Rev. Lett. 80, 2833 (1998).

[15] T. Nagatani, Rep. Prog. Phys. 65, 1331 (2002); D. Helbing, Rev. Mod. Phys. 73, 1067 (2001). 